\title{
Stereotactic Body Radiation Therapy for Renal Cell Carcinoma with Inferior Vena Cava Thrombus - Initial Experience Report and Literature Review
}

\author{
Yuval Freifeld $^{\mathrm{a}}$, Vitaly Margulis ${ }^{\mathrm{a}, \mathrm{c}}$, Solomon L. Woldu ${ }^{\mathrm{a}}$, Robert Timmerman ${ }^{\mathrm{b}, \mathrm{c}}$, \\ James Brugarolas ${ }^{\mathrm{c}, \mathrm{d}}$ and Raquibul Hannan ${ }^{\mathrm{b}, \mathrm{c}, *}$ \\ ${ }^{a}$ Department of Urology, University of Texas Southwestern Medical Center, Dallas, TX, USA \\ ${ }^{\mathrm{b}}$ Department of Radiation Oncology, University of Texas Southwestern Medical Center, Dallas, TX, USA \\ ${ }^{\mathrm{c}}$ Kidney Cancer Program, Simmons Comprehensive Cancer Center, University of Texas \\ Southwestern Medical Center, Dallas, TX, USA \\ ${ }^{\mathrm{d}}$ Division of Hematology/Oncology, Department of Internal Medicine, University of Texas Southwestern \\ Medical Center, Dallas, TX, USA
}

Received 20 September 2018

Accepted 8 January 2019

\begin{abstract}
.
Background: Renal cell carcinoma (RCC) with inferior vena cava thrombus (IVC-TT) represents a relatively infrequent presentation. Curative treatment includes extirpative surgery; however, this is associated with high rates of recurrence and complications. Stereotactic body radiation therapy (SBRT) has been used to treat metastatic RCC with good results. SBRT may be used as part of multimodal therapy to provide local control of IVC-TT.

Objective: We report our initial experience with SBRT to IVC-TT, including extended follow-up, and review the literature. Results: We report on two patients with level IV IVC-TT. Both had progressive disease while receiving systemic therapy and were eventually treated with SBRT to the IVC-TT, which showed good local control. Overall survival from the time of SBRT was 18 and 34 months, with no additional systemic therapy; one patient underwent additional SBRT and resection of metastatic sites.
\end{abstract}

Conclusions: SBRT to RCC IVC-TT may be considered in selected patients for local tumor control.

Keywords: RCC, SBRT, IVC, tumor thrombus, SABR, nephrectomy, stage III renal cancer, invasion, vascular migration, UTSW

\section{INTRODUCTION}

Kidney cancer is the 13th most common cancer worldwide, with renal cell carcinoma (RCC) account-

\footnotetext{
${ }^{*}$ Correspondence to: Raquibul Hannan, MD, PhD, Associate Professor, Department of Radiation Oncology, UT Southwestern Medical Center, 2280 Inwood Road, Dallas, TX 75390-9303, USA. Tel.: +1 (214) 645 7696; Fax: +1 (214) 645 7624; E-mail: Raquibul.Hannan@UTSouthwestern.edu.
}

ing for over $90 \%$ of all cases [1]. The expected incidence and mortality of RCC in the United States in 2018 is more than 65,000 new cases and more than 14,000 deaths [2]. A unique and often perilous feature of RCC is its ability to invade the venous system and form an inferior vena cava tumor thrombus (IVC-TT), which may extend as high as the right atrium [3]. At least $4-10 \%$ of patients are diagnosed with advanced disease characterized by IVC-TT [3, 4]. Although 
surgery is the only treatment with curative potential in RCC patients with IVC-TT, many patients may be poor surgical candidates, due to comorbidities and high risk of perioperative morbidity and mortality [5]. Expectant management of these patients is associated with poor survival [6]. Furthermore, sequelae such as pulmonary emboli, Budd-Chiari syndrome [7], and venous congestion may develop, further stress the need for additional treatment strategies.

Although RCC has been historically considered resistant to conventional radiation therapy, good control rates have been reported with the use of stereotactic ablative radiation therapy (SBRT) in patients with both localized and metastatic disease, although long term data are limited [8-10].

We report an update on our initial experience with SBRT to RCC IVC-TT, the first such experience reported in the literature, providing an extended follow-up [11] and a review of the literature.

\section{CASE DESCRIPTION}

Two patients with RCC and IVC-TT were treated at the University of Texas Southwestern (UTSW) Medical Center with SBRT aimed at IVC-TT between September 2012 and February 2013.

Case 1 - A 74-year old male initially presented with a $5-\mathrm{cm}$ right renal mass, a level IV IVC-TT (Fig. 1A), and suspected tumor pulmonary embolism. The patient underwent angio-embolization of the right kidney followed by radical nephrectomy and thrombectomy. Pathological analyses revealed a Fuhrman grade 3 clear cell RCC and a 15-cm IVC-TT invading the IVC wall with positive resection margins. Despite gross removal of the TT, shortly after the operation, a recurrent subdiaphragmatic IVC-TT was diagnosed (Fig. 1B). The patient was initially treated with sunitinib, yet the TT continued to progress. The patient received additional systemic therapy, which included everolimus, pazopanib, and axitinib, but the TT continued to progress with concern for extension to the right atrium. Surgical treatment was offered, but the patient refused. As an alternative, SBRT was delivered to the tumor thrombus with a total dose of $50 \mathrm{~Gy}$ in 5 fractions (Fig. 1C). No serious early or late adverse events were observed. Systemic therapy was discontinued, as it was poorly tolerated and of questionable efficacy (the patient had some lung nodules, but they had remained stable throughout the disease course). We previously reported a 24month follow-up of this patient with a reduction in size and enhancement of the tumor thrombus, despite no additional systemic therapy (Fig. 1D) [11]. After a follow-up of an additional 10 months, the patient developed left proximal femur and multiple liver metastases. The left femur metastasis was resected, and the area subsequently underwent radiation treatment (20 Gy in 5 fractions). Additional SBRT was administered to the (dominant) liver metastasis (single dose of $35 \mathrm{~Gy}$ ). Overall, the patient survived for 54 months from the time of initial diagnosis and for 34 months after initial IVC-TT SBRT with no systemic therapy. Over this span of time, the TT did not progress.

Case 2 - An 83-year old male patient was diagnosed with a large $(11 \mathrm{~cm})$ right renal mass, a level IV IVC-TT (Fig. 2A), lymphadenopathy and an osseous metastasis (T12). Biopsy revealed high grade papillary RCC. Because of comorbidities and poor medical condition, the patient could not undergo surgery and was instead treated with temsirolimus. However, retroperitoneal adenopathy and IVC-TT continued to progress (Fig. 2B), and the patient developed lower extremity edema. Because of concerns of further morbidity secondary to the IVC-TT, he was referred for SBRT. Treatment was planned for $45 \mathrm{~Gy}$ in 5 fractions (Fig. 2C-D). However, the patient decided to cease therapy after 4 sessions and to continue with palliative care only; at this point, systemic treatment was also stopped (after an overall treatment period of 4 months with temsirolimus). The patient survived for 18 more months without systemic therapy. Unfortunately, no scans are available to assess stability of the thrombus following SBRT.

\section{PROGNOSIS OF ADVANCED RCC WITH IVC-TT}

At the time of diagnosis, up to $30-50 \%$ of patients with IVC-TT present with metastatic disease [12-14]. Based on the Surveillance Epidemiology and End Results (SEER) database, Reese et al. evaluated the natural history of IVC-TT in patients who did not receive surgery, reporting a median survival of only 5 months and 29\% 1-year disease-specific survival (DSS) [6]. Although retrospective and prone to selection bias, these data point to the poor prognosis associated with untreated IVC-TT.

Surgical resection is the only current treatment with curative potential for RCC and IVC-TT. Despite radical nephrectomy and IVC thrombectomy, there is still an increased risk of recurrence [15, 16]. A review 

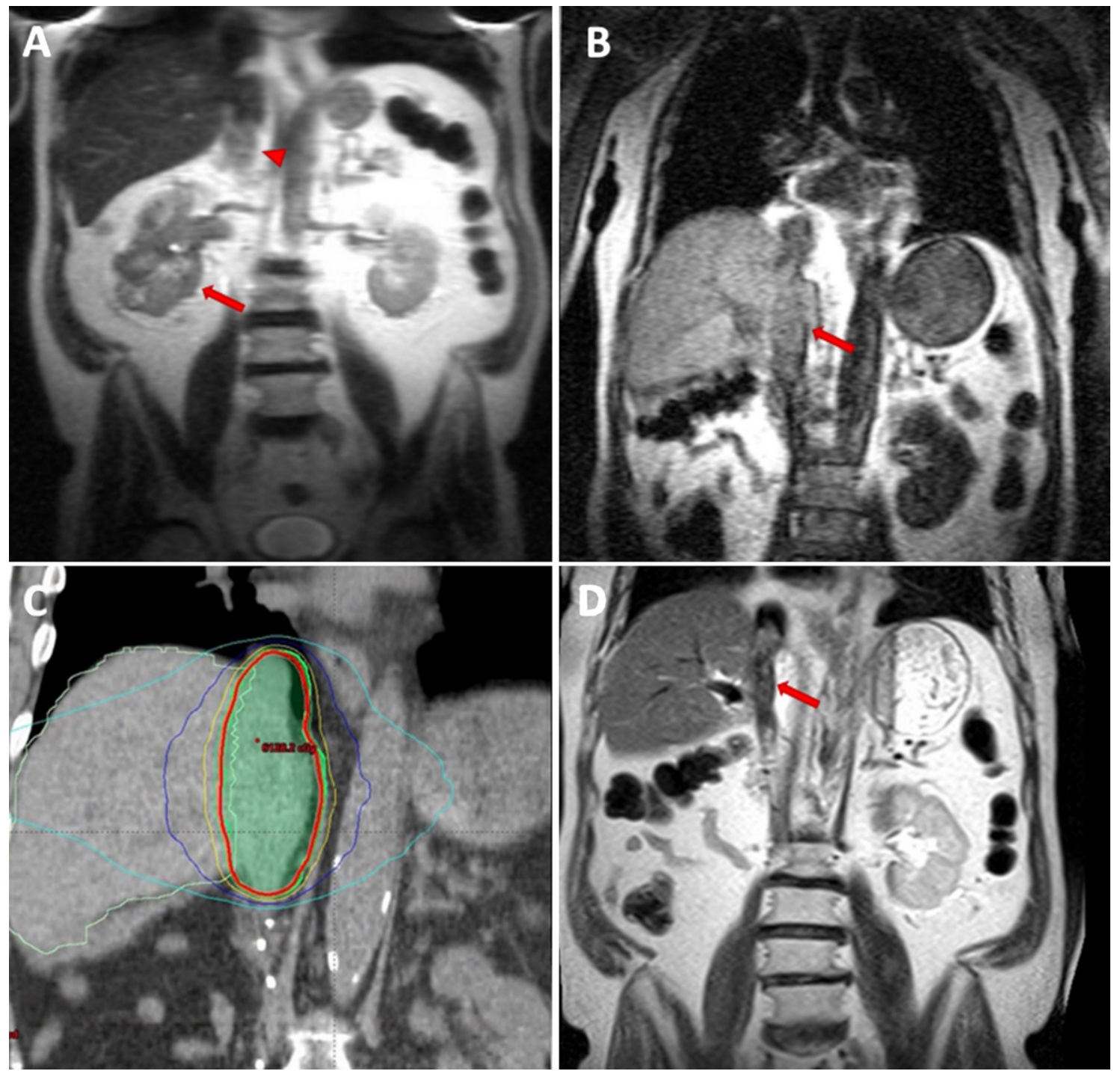

Fig. 1. MRI images of a 74 y/o man with right renal mass (arrow) and tumor thrombus (arrow head) extending to the right atrium (level IV) (A). After radical nephrectomy and thrombectomy, IVC tumor thrombus recurred (arrow) (B). SBRT ( $5 \times 10$ Gy) was delivered to the thrombus area-CT images demonstrate radiation planning (red contour representing the $95 \%$ iso-dose line) (C). Follow-up MRI, 16 months after completion of SBRT showing a decrease in IVC tumor thrombus size and enhancement (arrow) (D).

by Pouliot et al. reported a median survival of 38-116 months and a 5 -year DSS of $40-65 \%$ for patients with non-metastatic disease, and a median survival of 11-20 months and a 5-year DSS of 6.5-28\% for those with metastatic disease [12]. Specifically focusing on patients with high level thrombus, Haddad et al. reported a $49 \%$ 5-year cancer specific survival (CSS) and $42 \%$ overall survival (OS) for patients with non-metastatic disease. They recognized various risk factors associated with death due to RCC, including regional nodal metastasis, systemic metastasis, high grade tumors (grade 4), necrosis, and increased alkaline phosphatase [15]. Abel et al. developed a nomogram to predict recurrence and identified tumor diameter, body mass index, perioperative hemoglobin lower than normal limit, thrombus level, perinephric fat invasion, and non-clear cell histology as risk factors [16].

Various research groups have reported that overall survival is independent of thrombus level $[3,13]$. However, because higher level thrombi (III-IV) often require cardiopulmonary bypass or other advanced surgical techniques, those cases are associated with higher complication rates. Blute et al. reported 15\% 

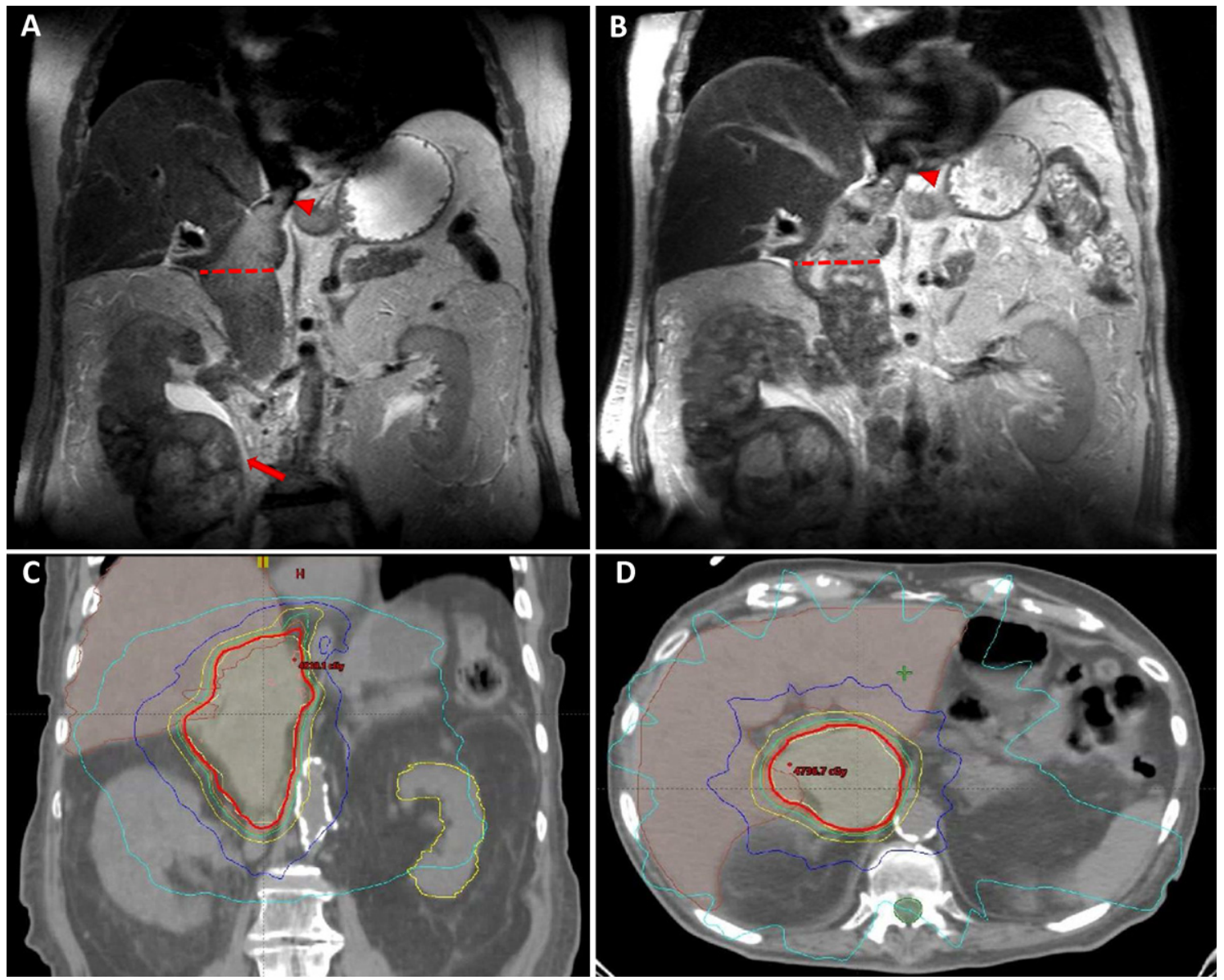

Fig. 2. MRI images of an 83 y/o man with right renal mass (arrow) and level IV tumor thrombus (arrow head), with a maximal diameter of $49 \mathrm{~mm}$ (dotted line) (A). Progressive increase in tumor thrombus size and enhancement, measuring $57 \mathrm{~mm}$ (dotted line and arrow head) (B). SBRT $(5 \times 9 \mathrm{~Gy})$ was planned covering the thrombus area - CT images demonstrate radiation planning (red contour representing the $95 \%$ iso-dose line) (C-D).

complication rates for level I-II thrombi compared to $30 \%$ for level IV [3]. Current multi-center reports have described similar rates [5, 17].

Furthermore, although surgical resection is the only curative option in these patients, postoperative morbidity after such procedures still reaches $30-35 \%$ [5]. Because many of these patients are elderly and may be at high surgical risk, other viable treatment options should be sought.

\section{SBRT FOR RCC}

Historically, RCC has been considered resistant to radiation therapy, but accumulating evidence suggests that RCC is sensitive to hypo-fractionated radiation therapy, such as SBRT. In vitro studies showed decreased RCC cell survival with radiation doses higher that 6 Gy per fraction [9]. After delivering SBRT with 16 Gy per fraction to mice with RCC, previous studies from our institution showed good local response rates with over $70 \%$ decrease in tumor size and no mitotic activity after tumor excision [18].

Accordingly, initial clinical reports suggested good control rates for both localized and metastatic disease using SBRT. Siva et al. systematically reviewed studies conducted on 126 patients treated with SBRT for localized disease, reporting estimated 2-year local control rates of $93 \%$. The rate of severe adverse events ( $\mathrm{AE} \geq$ grade 3 ) was $3.8 \%$ and the rate of minor $\mathrm{AE}$ was $21.4 \%$ [8]. Recent prospective trials including small cohorts of 15-30 patients and various regimens of SBRT, as high as $25 \mathrm{~Gy}$ per fraction, reported overall good local control rates ranging from $87 \%$ to $95 \%$; however, follow-up was limited [9]. 
Radiation therapy has been the therapy of choice for RCC metastasis in the central nervous system (CNS) and bones for many years. Recent data indicate local control rates of $84 \%$ for brain metastasis with stereotactic radiosurgery, which was also associated with improved overall survival $[19,20]$. Similar control rates were reported from UT Southwestern with a median follow-up of 2-years (86\%) [21]. Importantly, the survival of patients with and without brain metastases in our own small institutional series was comparable [21, 22]. Wang et al. described one of the largest retrospective cohorts of patients treated with SBRT for extracranial RCC metastasis, including 175 lesions from 84 patients [10]. Radiation was delivered as 40-60 Gy / 5 fractions, 30-54 Gy / 3 fractions, or $20-40 \mathrm{~Gy}$ in a single fraction. The local control rate was $91 \%$ after a median follow-up of 16.7 months. The authors found that lower recurrence risk was associated with previous systemic therapy ( $<2$ lines) and a biologically effective dose (BED) of $>98.7$ Gy. Only minor acute AEs (grade 1-2) were reported following $10.4 \%$ of treatments. However, 5 grade 3 late AEs ( $>3$ months following therapy) were reported, 2 of which were due to gastrointestinal bleeding that required surgery [10]. A recent report that focused on lung metastasis reported 1 and 3-year local control rates of $98 \%$ and $92 \%$, respectively, also suggesting that better control rates are associated with higher radiation doses $(\mathrm{BED} \geq 120 \mathrm{~Gy}, p=0.054)$ [23].

\section{SBRT FOR TUMOR THROMBUS}

While the two cases we report are the first published in the kidney cancer literature, a few groups have reported the effectiveness of SBRT in treating tumor-related thrombus in hepatocellular carcinoma. $\mathrm{Xi}$ et al. used SBRT to treat advanced hepatocellular carcinoma with invasion of the portal vein (PV-TT) or IVC in 41 patients. The SBRT median dose was $36 \mathrm{~Gy}$ in 6 fractions. After a median follow-up of 10 months, only $8 \%$ of treated tumors progressed with $36 \%$ having a complete response [24]. Similarly, Matsu et al. reported high tumor response rates for hepatocellular carcinoma-related tumor thrombus of about $70 \%$ [25].

We described the first two cases of RCC-related IVC-TT treated with SBRT with extended follow-up. So far, our initial experience has shown good control rates with minimal AE. Survival after SBRT ranged from 18 to 36 months, both with high level IVCTT and symptomatic disease despite no systemic therapy. These results are similar to those observed after surgical resection and better than those expected with conservative management, suggesting SBRT is a viable treatment option in selected patients.

Based on our experience and available data, we have initiated a phase II trial of neoadjuvant SBRT for IVC-TT (NCT02473536) to reduce systemic recurrence. Initial results from the safety lead-in phase of this trial on 6 patients were recently presented [26]. SBRT was delivered with a total dose of $40 \mathrm{~Gy}$ divided into 5 fractions to the IVC-TT, followed by nephrectomy and thrombectomy. No serious $\mathrm{AE}$ ( $\geq$ grade 3 ) were reported, and only mild AE grade 1-2 were associated with radiation therapy [26]. These data suggest that neoadjuvant SBRT to IVC-TT is feasible and safe.

In our opinion, SBRT treatment to IVC-TT should be considered in several settings. First, in patients with an isolated IVC-TT recurrence who are not optimal surgical candidates or who would prefer a less morbid intervention than a second surgery. In these patients, SBRT may obviate the need for systemic therapy while offering good local control rates and avoiding toxicity from systemic therapy.

Second, SBRT to an IVC-TT may also be considered in patients with localized disease who are not surgical candidates and for whom SBRT may be administered to both the IVC-TT and the primary tumor. Third, SBRT to an IVC-TT may also be considered in patients with metastatic disease and IVC-TT, who may not be optimal surgical candidates due to the extent/bulkiness of the metastases, or due to comorbidities. In these patients a trial of systemic therapy may be warranted. However, if there are concerns about the development of Budd-Chiari syndrome or isolated progression of the TT, SBRT may be fitting.

While the CARMENA trial [27] suggests that there may be limited benefit to cytoreductive nephrectomy, we still believe that patients with high level tumor thrombi and no bulky metastases may benefit from surgery. In the setting of bulkier metastatic disease, systemic therapy up front may be reasonable.

Finally, SBRT may also be considered as a single treatment modality in the setting of palliative treatment for advanced IVC-TT.

\section{CONCLUSIONS}

SBRT may be considered as a treatment option for managing RCC, as indicated by recent evidence accumulated on treatment of both primary and metastatic 
disease. We use SBRT routinely for the treatment of brain and bone metastasis. Based on our experience, SBRT to IVC-TT is feasible and safe, and may be a viable treatment option for selected patients. In the future, SBRT may also be part of multimodal treatment, potentiating response rates to systemic therapy and improving disease-free survival in patients undergoing surgery.

\section{ACKNOWLEDGMENTS}

This work was supported by the American Cancer Society RSG-16-004-01-CCE to RH and National Institutes of Health (T32 CA136515 Ruth L. Kirschstein Institutional National Research Award to S.L.W. and P50CA196516 to J.B.).

\section{CONFLICT OF INTERESTS}

The authors have no conflict of interest to report.

\section{REFERENCES}

[1] Ljungberg B, Campbell SC, Cho HY, Jacqmin D, Lee JE, Weikert S, Kiemeney LA. The Epidemiology of Renal Cell Carcinoma. European Urology. 2011;60(4):615-21.

[2] Siegel RL, Miller KD, Jemal A. Cancer statistics, 2018. CA: A Cancer Journal for Clinicians. 2018;68(1):7-30.

[3] Blute Michael L, Leibovich Bradley C, Lohse Christine M, Cheville John C, Zincke H. The Mayo Clinic experience with surgical management, complications and outcome for patients with renal cell carcinoma and venous tumour thrombus. BJU International. 2004;94(1):33-41.

[4] Kim HL, Zisman A, Han K-R, Figlin RA, Belldegrun AS. Prognostic Significance of Venous Thrombus in Renal Cell Carcinoma. Are Renal Vein and Inferior Vena Cava Involvement Different? The Journal of Urology. 2004;171(2, Part 1):588-91.

[5] Haddad AQ, Leibovich BC, Abel EJ, Luo J-H, Krabbe L-M, Thompson RH, Heckman JE, Merrill MM, Gayed BA, Sagalowsky AI, Boorjian SA, Wood CG, Margulis V. Preoperative multivariable prognostic models for prediction of survival and major complications following surgical resection of renal cell carcinoma with suprahepatic caval tumor thrombus. Urologic Oncology: Seminars and Original Investigations. 2015;33(9):388.e1-.e9.

[6] Reese AC, Whitson JM, Meng MV. Natural history of untreated renal cell carcinoma with venous tumor thrombus. Urologic Oncology: Seminars and Original Investigations. 2013;31(7):1305-9.

[7] Shih K-L, Yen H-H, Su W-W, Soon M-S, Hsia C-H, Lin YM. Fulminant Budd-Chiari syndrome caused by renal cell carcinoma with hepatic vein invasion: Report of a case. European Journal of Gastroenterology \& Hepatology. 2009;21(2).
[8] Siva S, Pham D, Gill S, Corcoran Niall M, Foroudi F. A systematic review of stereotactic radiotherapy ablation for primary renal cell carcinoma. BJU International. 2012;110(11b):E737-E43.

[9] Dengina N, Tsimafeyeu I, Mitin T. Current Role of Radiotherapy for Renal-Cell Carcinoma: Review. Clinical Genitourinary Cancer. 2017;15(2):183-7.

[10] Wang CJ, Christie A, Lin M-H, Jung M, Weix D, Huelsmann L, Kuhn K, Meyer J, Desai N, Kim DWN, Pedrosa I, Margulis V, Cadeddu J, Sagalowsky A, Gahan J, Laine A, Xie X-J, Choy H, Brugarolas J, Timmerman R, Hannan R. Safety and Efficacy of Stereotactic Ablative Radiation Therapy for Renal Cell Carcinoma Extracranial Metastases. International journal of radiation oncology, biology, physics. 2017;98(1):91-100.

[11] Hannan R, Margulis V, Chun SG, Cannon N, Kim DWN, Abdulrahman RE, Sagalowsky A, Pedrosa I, Choy H, Brugarolas J, Timmerman RD. Stereotactic radiation therapy of renal cancer inferior vena cava tumor thrombus. Cancer Biology \& Therapy. 2015;16(5):657-61.

[12] Pouliot F, Shuch B, LaRochelle JC, Pantuck A, Belldegrun AS. Contemporary Management of Renal Tumors With Venous Tumor Thrombus. The Journal of Urology. 2010;184(3):833-41.

[13] Wagner B, Patard J-J, Méjean A, Bensalah K, Verhoest G, Zigeuner R, Ficarra V, Tostain J, Mulders P, Chautard D, Descotes J-L, de la Taille A, Salomon L, Prayer-Galetti T, Cindolo L, Valéri A, Meyer N, Jacqmin D, Lang H. Prognostic Value of Renal Vein and Inferior Vena Cava Involvement in Renal Cell Carcinoma. European Urology. 2009;55(2):452-60.

[14] Abel EJ, Spiess PE, Margulis V, Master VA, Mann M, Zargar-Shoshtari K, Borregales LD, Sexton WJ, Patil D, Matin SF, Wood CG, Karam JA. Cytoreductive Nephrectomy for Renal Cell Carcinoma with Venous Tumor Thrombus. The Journal of Urology. 2017;198(2):281-8.

[15] Haddad AQ, Wood CG, Abel EJ, Krabbe L-M, Darwish OM, Thompson RH, Heckman JE, Merril MM, Gayed BA, Sagalowsky AI, Boorjian SA, Margulis V, Leibovich BC. Oncologic Outcomes Following Surgical Resection of Renal Cell Carcinoma with Inferior Vena Caval Thrombus Extending Above the Hepatic Veins: A Contemporary Multicenter Cohort. The Journal of Urology. 2014;192(4):1050-6.

[16] Abel EJ, Masterson TA, Karam JA, Master VA, Margulis V, Hutchinson R, Lorentz CA, Bloom E, Bauman TM, Wood CG, Blute ML. Predictive Nomogram for Recurrence following Surgery for Nonmetastatic Renal Cell Cancer with Tumor Thrombus. The Journal of Urology. 2017;198(4):810-6.

[17] Abel EJ, Thompson RH, Margulis V, Heckman JE, Merril MM, Darwish OM, Krabbe L-M, Boorjian SA, Leibovich BC, Wood CG. Perioperative Outcomes Following Surgical Resection of Renal Cell Carcinoma with Inferior Vena Cava Thrombus Extending Above the Hepatic Veins: A Contemporary Multicenter Experience. European Urology. 2014;66(3):584-92.

[18] Walsh L, Stanfield JL, Cho LC, Chang C-h, Forster K, Kabbani W, Cadeddu JA, Hsieh J-T, Choy H, Timmerman R, Lotan Y. Efficacy of Ablative High-Dose-per-Fraction Radiation for Implanted Human Renal Cell Cancer in a Nude Mouse Model. European Urology. 2006;50(4):795-800.

[19] Kim WH, Kim DG, Han JH, Paek SH, Chung H-T, Park C-K, Kim C-Y, Kim YH, Kim JW, Jung H-W. Early Significant Tumor Volume Reduction After Radiosurgery in 
Brain Metastases From Renal Cell Carcinoma Results in Long-Term Survival. International Journal of Radiation Oncology*Biology*Physics. 2012;82(5):1749-55.

[20] Ippen FM, Mahadevan A, Wong ET, Uhlmann EJ, Sengupta S, Kasper EM. Stereotactic Radiosurgery for Renal Cancer Brain Metastasis: Prognostic Factors and the Role of Whole-Brain Radiation and Surgical Resection. Journal of Oncology. 2015;2015:636918.

[21] Wardak Z, Christie A, Bowman A, Stojadinovic S, Nedzi L, Barnett S, Patel T, Mickey B, Whitworth T, Hannan R, Brugarolas J, Timmerman R. Stereotactic Radiosurgery for Multiple Brain Metastases from Renal Cell Carcinoma. Clinical Genitourinary Cancer.

[22] Bowman IA, Bent A, Le T, Christie A, Wardak Z, Arriaga Y, Courtney K, Hammers H, Barnett S, Mickey B, Patel T, Whitworth T, Stojadinovic S, Hannan R, Nedzi L, Timmerman R, Brugarolas J. Improved Survival Outcomes for Kidney Cancer Patients With Brain Metastases. Clinical Genitourinary Cancer. 2018.

[23] Hoerner-Rieber J, Duma M, Blanck O, Hildebrandt G, Wittig A, Lohaus F, Flentje M, Mantel F, Krempien R, Eble MJ, Kahl KH, Boda-Heggemann J, Rieken S, Guckenberger M. Stereotactic body radiotherapy (SBRT) for pulmonary metastases from renal cell carcinoma-a multicenter analysis of the German working group "Stereotactic Radiotherapy”. Journal of Thoracic Disease. 2017;9(11):4512-22.

[24] Xi M, Zhang L, Zhao L, Li Q-Q, Guo S-P, Feng Z-Z, Deng X-W, Huang X-Y, Liu M-Z. Effectiveness of Stereotactic Body Radiotherapy for Hepatocellular Carcinoma with Portal Vein and/or Inferior Vena Cava Tumor Thrombosis. PLoS ONE. 2013;8(5):e63864.
[25] Matsuo Y, Yoshida K, Nishimura H, Ejima Y, Miyawaki D, Uezono H, Ishihara T, Mayahara H, Fukumoto T, Ku Y, Yamaguchi M, Sugimoto K, Sasaki R. Efficacy of stereotactic body radiotherapy for hepatocellular carcinoma with portal vein tumor thrombosis/inferior vena cava tumor thrombosis: Evaluation by comparison with conventional three-dimensional conformal radiotherapy. Journal of Radiation Research. 2016;57(5):512-23.

[26] Freifeld Y, Hannan R, Woldu S, Bagrodia A, Gahan J, Timmerman R, Mohamad O, Laine A, Desai N, Brugarolas J, Margulis V. LBA28 SAFETY LEAD-IN OF A PHASE II TRIAL OF NEO-ADJUVANT SABR FOR IVC TUMOR THROMBUS IN RCC. The Journal of Urology. 2018;199(4):e1168.

[27] Méjean A, Ravaud A, Thezenas S, Colas S, Beauval J-B, Bensalah K, Geoffrois L, Thiery-Vuillemin A, Cormier L, Lang H, Guy L, Gravis G, Rolland F, Linassier C, Lechevallier E, Beisland C, Aitchison M, Oudard S, Patard J-J, Theodore C, Chevreau C, Laguerre B, Hubert J, GrossGoupil M, Bernhard J-C, Albiges L, Timsit M-O, Lebret T, Escudier B. Sunitinib Alone or after Nephrectomy in Metastatic Renal-Cell Carcinoma. New England Journal of Medicine. 2018;379(5):417-27. 